\title{
Minimalinvasive Chirurgie bei Malignomen des Gastrointestinaltrakts: Kolon - Kontra-Position
}

\author{
Philipp Lux Klaus Weber Werner Hohenberger \\ Chirurgische Klinik, Universitätsklinikum Erlangen, Deutschland
}

Schlüsselwörter

Chirurgie, laparoskopische $\cdot$ Kolonkarzinom

\section{Zusammenfassung}

Hintergrund: Darstellung der aktuellen Studienlage hinsichtlich des Vergleichs offener versus laparoskopischer Chirurgie bei Kolonkarzinomen. Methoden: Selektive Literaturrecherche und Daten aus dem Erlanger Register kolorektaler Karzinome. Ergebnisse: Die laparoskopische Resektion des Kolonkarzinoms kann bei entsprechender Expertise des Operateurs und geeigneter Selektion mit gleichen onkologischen Ergebnissen im Vergleich zur offenen Chirurgie durchgeführt werden. Schlussfolgerungen: In Zukunft müssen die Begriffe "Expertise» und "Selektion" genauer definiert werden, um die Rate an Konversionen mit den damit verbundenen schlechteren onkologischen Ergebnissen zu minimieren. Grundsätzlich muss sich jedoch die Qualität der laparoskopischen Chirurgie an derjenigen der optimierten offenen Vorgehensweise orientieren.

\section{Einleitung}

In der aktuell fertiggestellten S3-Leitlinie «Kolorektales Karzinom» [1] wird folgende Empfehlung zum Stellenwert der laparoskopischen Operation beim kolorektalen Karzinom ausgesprochen: «Die laparoskopische Resektion des Kolon- und Rektumkarzinoms kann bei entsprechender Expertise des Operateurs und geeigneter Selektion mit gleichen onkologischen Ergebnissen im Vergleich zur offenen Op-Technik durchgeführt werden (Level of Evidence: 1a, Empfehlungsgrad A).»

Diese Leitlinien-Empfehlung basiert unter anderem auf den Ergebnissen eines von Kuhry et al. [2] verfassten Cochrane-Reviews und auf kürzlich publizierten Langzeitergebnissen der britischen CLASICC-Studie [3].

\section{KARGER \\ Fax +497614520714 \\ Information@Karger.com}

www.karger.com (c) 2013 S. Karger GmbH, Freiburg

1662-6664/13/0296-0388\$38.00/0

Accessible online at:

www.karger.com/vim
Keywords

Laparoscopic surgery · Colon cancer

\section{Summary}

Minimally Invasive Surgery for Malignancies of the Gastrointestinal Tract: Colon - Contra Position

Background: Presentation of the current situation regarding studies comparing open versus laparoscopic surgery for colon cancer. Methods: Selective literature review and data from the Erlangen Registry of Colorectal Carcinomas. Results: With appropriate expertise of the surgeon and appropriate selection, laparoscopic resection of colon cancer can be performed with the same oncological results as compared to open surgery. Conclusions: In the future, the terms 'expert' and 'selection' must be defined more precisely in order to minimize the rate of conversions associated with poorer oncological results. Basically, the quality of laparoscopic surgery must be based on that of the optimized open procedure.
Eine klare «Kontra-Position» gibt es damit grundsätzlich nicht mehr. Der bisherige Passus in den Leitlinien von 2004 hatte der laparoskopischen Operation eher noch einen experimentellen Stellenwert zuerkannt [4]: «Die Ergebnisse der laparoskopischen Tumorresektion sind derzeit wegen fehlender onkologischer Langzeitergebnisse nicht abschließend zu beurteilen, sodass dieses Verfahren nur im Rahmen von qualifizierten Studien mit langfristiger Verlaufbeobachtung zur Anwendung kommen sollte. Empfehlungsgrad: A, Evidenzstärke: 2a, mehrheitliche Zustimmung.»

Nunmehr gilt es zu entscheiden, was «Expertise des Operateurs» und «geeignete Selektion» bedeuten.

Expertise (besondere Erfahrung und sachgerechter Umgang) kann man im vorliegenden Zusammenhang an der An- 
zahl der durchgeführten Operationen, mehr aber noch an den Konversionsraten und intra- sowie postoperativen Komplikationen, bei onkologischen Eingriffen aber auch noch an den spezifischen Parametern wie Qualität der mesokolischen Schichten, Lokalrezidivraten und Überlebensdaten festmachen.

Selektion setzt diese Parameter noch in das Verhältnis zu tumorspezifischen Charakteristika (Lokalisation, Tumorkategorien) oder besonderen Umständen (Notfälle, Body-MassIndex (BMI)).

\section{Vorgehensweise}

Es erfolgte eine selektive Literaturrecherche unter besonderer Berücksichtigung:

- der systematischen Recherche für die neue S3-Leitlinie Kolorektales Karzinom (bis März 2011),

- des Cochrane-Reviews von Kuhry et al. [2] von 2012 (Daten bis 1/2008),

- weiterer eigener Handsuche (bis 2/2013),

- Erlanger Daten (Erlanger Register kolorektaler Karzinome (ERCRC), 1453 konsekutive Patienten mit Kolonkarzinomen, die zwischen 1978 und 2004 offen R0-reseziert wurden (publiziert in [5]), bzw. 1705 konsekutive Patienten mit Kolonkarzinomen, die zwischen 1978 und 2005 offen operiert wurden (publiziert in [6])).

\section{Ergebnisse}

\section{Expertise}

\section{Lernkurve}

Nach Drolet et al. [7] kann als «High-Volume»-Chirurg gelten, wer mindestens 10 Kolonkarzinome pro Jahr überhaupt operiert. Daraus resultiert im Vergleich $\mathrm{zu}$ «LowVolume»-Chirurgen ( $<5$ Fälle pro Jahr) eine signifikante Reduktion der Morbidität und Mortalität.

In einer deutschen Studie, die in 341 Kliniken insgesamt 21721 Kolonkarzinompatienten im Zeitraum von 2000 bis 2003 umfasste, zeigte sich, dass in $90 \%$ der deutschen Kliniken weniger als 10 laparoskopische Kolonresektionen pro Jahr durchführt wurden [8].

In der internationalen Literatur wird als erforderliche Lernkurve für laparoskopische kolorektale Resektionen häufig eine durchschnittliche Zahl von 30-70 Fällen pro Operateur angegeben $[9,10]$. Daher würde ein Chirurg, wenn man nur auf Kolonkarzinome reflektiert und zudem die ganz überwiegend in Deutschland pro Klinik operierten Fälle berücksichtigt, mehr als 5 Jahre benötigen, um die durchschnittlich erforderliche jährliche Fallzahl zu erreichen, um die laparoskopische Kolonresektion mit adäquater Qualität beherrschen zu können [11].

Einzelne Autoren gehen hinsichtlich der für eine adäquate Lernkurve erfordlichen Fallzahl sogar deutlich weiter: Bött- ger et al. [12] fordern, dass «von einem in der offenen kolorektalen Chirurgie erfahrenen Operateur mit Anfangserfahrung in der laparoskopischen Chirurgie zirka 35 laparoskopische Rektumresektionen und 200 laparoskopische Kolonresektionen notwendig sind, bis Selektionsrate, Operationszeit, allgemeine und chirurgische Komplikationen ein Plateau erreicht haben».

\section{Konversionsraten}

Insbesondere bei Kliniken mit geringen kolorektalen Fallzahlen war in der Vergangenheit die Konversionsrate sehr hoch. Betrachtet man die oben bereits erwähnten Daten von Kube et al. [8], zeigt sich, dass $90 \%$ der Kliniken in Deutschland weniger als 10 laparoskopische Kolonresektionen pro Jahr durchführten und dabei eine Konversionsrate von 25\% zu verzeichnen war. Bei 10-19 laparoskopischen Kolonresektionen pro Jahr (8,2\% der Kliniken) sank die Konversionsrate bereits deutlich auf $17 \%$. Nur $1,4 \%$ der Kliniken $(n=2)$ führten mehr als 20 derartige Eingriffe pro Jahr durch, wobei nur in $3,6 \%$ der Fälle zur offenen Operation konvertiert werden musste. Auch im Rahmen der internationalen Studien zum Vergleich von laparoskopischer mit offener Operationstechnik beim kolorektalen Karzinom wurden hohe Konversionsraten beschrieben (Clinical Outcomes of Surgical Therapy (COST): 21\%, Colon Carcinoma Laparoscopic or Open Resection (COLOR): 17\%, Conventional Versus LaparoscopicAssisted Surgery in Colorectal Cancer (CLASICC): 25\%).

Diese hohen Konversionsraten sind insofern inakzeptabel, als im Langzeit-Follow-up sowohl der CLASICC-Studie [3] als auch der COST-Studie [13] gezeigt wurde, dass Patienten, bei denen von laparoskopischer zur offenen Operation konvertiert wurde, ein signifikant schlechteres Gesamtüberleben hatten.

\section{Lokalrezidive und Überleben}

In keiner der anerkannten randomiserten kontrollierten Studien (COST, CLASICC, COLOR) konnte im LangzeitFollow-up ein Unterschied bezüglich laparoskopischer oder offener Chirurgie festgestellt werden, weder für die Rate an Lokalrezidiven noch für die Überlebensraten (Gesamtüberleben, krankheitsfreies Überleben) [2, 13, 14].

Ebenfalls wurde kein signifikanter Unterschied in der Entwicklung von Fernmetastasen bei Patienten mit kolorektalem Karzinom beim Vergleich von laparoskopischer und offener Chirurgie gefunden [2].

Ein wichtiger Aspekt zur Beurteilung dieser onkologischen Ergebnisse ist jedoch die Tatsache, dass die Patienten insbesondere in den laparoskopischen Armen der oben zitierten randomisierten kontrollierten Studien teilweise erheblichen Selektionskriterien unterworfen waren und sich trotz dieser Selektion noch hohe Konversionsraten ergaben. Auf diese soll im Abschnitt «Selektion» näher eingegangen werden.

Wie bereits oben erwähnt, wurde jedoch deutlich, dass eine Konversion von offener zur laparoskopischen Chirurgie zu 
Tab. 1. Vergleich onkologischer Ergebnisse (modifiziert nach $[6,13])$

\begin{tabular}{|c|c|c|c|c|}
\hline Studie (RCT) & $\begin{array}{l}\text { Follow-up, Monate, } \\
\text { Median (Range) }\end{array}$ & $\begin{array}{l}\text { 5-Jahres-Gesamtüberleben, } \\
\%\end{array}$ & $\begin{array}{l}\text { 5-Jahres-krankheitsfreies } \\
\text { Überleben, \% }\end{array}$ & $\begin{array}{l}\text { Lokalrezidive, } \\
\%\end{array}$ \\
\hline \multirow[t]{2}{*}{ COST (2007) } & \multirow[t]{2}{*}{$84(60-120)$} & LC 76,4 & LC 69,2 & LC 2,3 \\
\hline & & OC 74,6 & OC 68,4 & OC 2,6 \\
\hline \multirow[t]{2}{*}{ Lacy et al. (2008) } & \multirow[t]{2}{*}{$95(77-133)$} & LC 64 & LC 84 & LC 7,5 \\
\hline & & OC 51 & OC 73 & OC 13,7 \\
\hline \multirow[t]{2}{*}{ CLASICC (2010) } & \multirow[t]{2}{*}{$69,8(57-92)$} & LC 55,7 & LC 57,6 & LC 10,8 \\
\hline & & OC 62,7 & OC 64,0 & OC 8,7 \\
\hline \multirow[t]{2}{*}{ Braga et al. (2010) } & \multirow[t]{2}{*}{$73(48-106)$} & LC 72 & LC 63 & LC 0,7 \\
\hline & & OC 66 & OC 63 & OC 2,2 \\
\hline \multirow{2}{*}{$\begin{array}{c}\text { Erlangen (Merkel } \\
\text { et al., 2012) }\end{array}$} & \multirow[t]{2}{*}{$108(0-384)$} & OC 73,5 (vor 1995) & OC 67,9 (vor 1995) & OC 4,8 \\
\hline & & OC 78,4 (nach 1995) & OC 70,7 (nach 1995) & \\
\hline
\end{tabular}

RCT = Randomisierte kontrollierte Studie; LC = laparoskopische Chirurgie; OC = offene Chirurgie. einer signifikanten Verschlechterung der 5-Jahres-Überlebensraten der konvertierten Patienten führte [3].

Interessanterweise zeigte sich in den erst kürzlich publizierten partiellen 10-Jahres-Daten der CLASICC-Studie [15] ein signifikanter Unterschied im krankheitsfreien Überleben zwischen rechts- und linksseitigen Kolonkarzinomen (Hazard Ratio (HR) 0,68, 95\%-Konfidenzintervall (CI) 0,48-0,97; p = $0,031)$ und ein nicht signifikanter Trend zu erhöhten Lokalrezidiven bei den rechtsseitigen Kolonkarzinomem im Vergleich mit linksseitigen und Sigma-Karzinomen (5,2 vs. 14,7\%).

Auch stadienabhängig wurde kein signifikanter Unterschied zwischen den beiden Techniken (laparoskopische Kolonresektion vs. offene Resektion) festgestellt [3]. Allerdings zeigte sich in den neuesten Daten der CLASICC-Studie ein nicht signifikanter Trend hin zu einer verbesserten Überlebensrate nach einer offenen Operation bei Patienten mit Union for International Cancer Control(UICC)-Stadium III bei Kolonkarzinomen [15].

Hierzu sollte erwähnt werden, dass weder eine komplette mesokolische Exzision (CME) [16] noch eine Ligatur der zentralen Gefäße (CVL) im laparoskopischen Arm der CLASICC-Studie angewendet wurden.

Quirke [17] konnte anhand der CLASICC-Daten analog zu den Rektumkarzinomen auch für Kolonkarzinome zeigen, dass die Qualität der Präparate hinsichtlich der Intaktheit der mesokolischen Schichten wesentlichen Einfluss auf die onokolischen Langzeitergebnisse hat. Bei schlechter Qualität der Resektate (Einrisse bis auf die Muscularis-propria-Schicht) wurden Lokalrezidivraten bis $22 \%$ beobachtet. West et al. [18] beschrieben insbesondere bei Kolonkarzinomen im Stadium III deutlich verbesserte Überlebensraten, wenn eine qualitativ hochwertige Chirurgie unter Berücksichtigung der mesokolischen Schichten (CME) durchgeführt wurde. Hier zeigte sich ein Vorteil bei den 5-Jahres-Überlebensraten um bis zu $27 \%$.

Bei keiner der bisherigen randomisierten Studien zum Vergleich von laparoskopischer mit offener Chirurgie bei Kolonkarzinomen erfolgte eine standardisierte chirurgische Qualitätssicherung mittels Bewertung der Qualität der Resektate.
In der aktuell fertiggestellten S3-Leitlinie «Kolorektales Karzinom» wird analog zu der bisher schon etablierten Qualitätsbewertung der Resektatqualität der Rektumkarzinome nun auch eine Qualitätsbewertung der Resektatqualität der Kolonkarzinome eingeführt. Verbunden damit wird empfohlen, alle Kolonresektionen künftig als CME durchzuführen, da gezeigt werden konnte, dass diese zu einer signifikant besseren Resektatqualität, verbunden mit daraus resultierenden besseren onkologischen Langzeitergebnissen, führt [19].

Bisher gibt es keine Ergebnisse von kontrolliert-randomisierten Studien zum Vergleich von laparoskopischer mit offener CME. Allerdings zeigte eine Studie einer griechischen Arbeitsgruppe [20], dass sowohl bei rechtsseitigen als auch bei linksseitigen laparoskopischen Hemikolektomien mittels CME eine vergleichbar gute Resektatqualität erzielt werden kann. Lediglich bei Transversumkarzinomen war die Qualität der laparoskopischen Resektate schlechter. Dies zeigte sich in einer kürzeren Länge der Strecke zwischen zentraler Gefäßligatur und Tumor (offen 11,67 cm vs. laparoskopisch 8,72 cm; $\mathrm{p}=0,049$ ) und einer geringeren Lymphknotenausbeute (offen 46,33 vs. laparoskopisch 39,33; $\mathrm{p}=0,033)$.

Im Vergleich mit den Daten der meisten kontrolliert-randomisierten Studien mit ausreichend hoher Fallzahl konnten wir zeigen, dass durch Optimierung der Technik der offenen Kolonresektionen mittels CME und CVL eine deutliche Verbesserung der 5-Jahres-Überlebensraten und eine Reduktion der Rate an Lokalrezidiven zu erzielen ist (Tab. 1).

\section{Postoperative Komplikationen}

Im Rahmen der im Cochrane-Review von Kuhry et al. [2] untersuchten Studien zeigten sich keine signifikanten Unterschiede im Auftreten von Narbenhernien und Re-Operationen bei Verwachsungen zwischen laparoskopisch assistierter und offener Chirurgie.

In einem Cochrane-Review von 2005 [21] wurde gezeigt, dass die Intensität der postoperativen Schmerzen geringer und die Dauer des postoperativen Ileus nach laparoskopischen kolorektalen Resektionen kürzer war als nach offener Chirurgie. Obgleich laparoskopische kolorektale Resektionen 
längere Operationszeiten bedingen, sprechen zahlreiche randomisierte Studien dafür, dass im Kurzzeitverlauf die perioperative chirurgische Morbidität niedriger ist als nach konventioneller Operation, bei unveränderter Gesamtmorbidität und Letalität [14, 21, 22].

Auch neuere Metaanalysen [13,14] zeigen, dass bei den laparoskopischen Resektionen zwar die Operationsdauer länger, dafür der Krankenhausaufenthalt jedoch kürzer ist. Der intraoperative Blutverlust ist bei den laparoskopischen Resektionen signifikant geringer. Die Rate an Anastomoseninsuffizienzen ist bei beiden Verfahren gleich.

\section{Lebensqualität}

Die Langzeitdaten der schwedischen COLOR-Studie zeigten, dass die Verbesserung der postoperativen Lebensqualität nach laparoskopischer Resektion von Kolonkarzinomen nur auf den ersten postoperativen Monat beschränkt war [23].

In einer anderen Studie [24] zeigte sich eine Verbesserung der Lebensqualität zugunsten der laparoskopischen Gruppe für die ersten 6 Monate nach der Operation; danach war auch hier kein Unterschied mehr feststellbar.

Im Rahmen der CLASICC-Studie fanden sich allerdings keine signifikanten Verbesserungen der kurzfristigen Lebensqualität [13].

\section{Selektion}

Basis einer vernünftigen Selektion ist nach erfolgter koloskopischer Lokalisation und histologischer Sicherung gegebenenfalls auch die Durchführung einer erweiterten Diagnostik, wenn in der Basisdiagnostik (vor allem Sonographie des Abdomens) der Verdacht auf ein organüberschreitendes Wachstum besteht. Durch verbesserte präoperative Bildgebung (Computertomographie (CT)) sind damit wahrscheinlich niedrigere Konversionsraten möglich. Die Durchführung einer CT des Abdomens als Standarddiagnostik bei jedem neu diagnostizierten Kolonkarzinom wird jedoch auch in der aktuellen Leitlinienversion nach wie vor nicht routinemäßig empfohlen.

Im eigenen, nicht selektierten Krankengut ([5] bzw. Erlanger Krebsregister (Tab. 2)) ist in 18\% der Fälle mit Transversumkarzinomen (die Flexuren eingeschlossen), in 14\% mit organüberschreitend wachsenden Karzinomen und in 9\% mit Notfalloperationen zu rechnen. Diese Patienten werden ebenso wie Patienten mit Mehrfachkarzinomen von der laparoskopischen Operation in der Regel ausgeschlossen.

Betrachtet man beispielsweise die Einschlusskriterien der amerikanischen COST-Studie, entsprachen die damit erfassten Patienten nur knapp 40\% aller auslesefrei erfassten konsekutiven Fälle bei Kolonkarzinomen der Erlanger Klinik. Darüber hinaus wurde in der COST-Studie dann noch in $21 \%$ der Fälle vom laparoskopischen zum offenen Vorgehen konvertiert [25].
Tab. 2. Erlanger Krankengut $(\mathrm{n}=1542)$ (modifiziert nach $[5])$

\begin{tabular}{|c|c|c|}
\hline & Anzahl & Prozentsatz \\
\hline \multicolumn{3}{|l|}{ Tumorlokalisation } \\
\hline Colon sigmoideum & 813 & 52,7 \\
\hline Colon descendens & 90 & 5,8 \\
\hline Linke Kolonflexur & 71 & 4,6 \\
\hline Colon transversum & 137 & 8,9 \\
\hline Rechte Kolonflexur & 75 & 4,9 \\
\hline Colon ascendens & 224 & 14,5 \\
\hline Zoekum & 132 & 8,6 \\
\hline \multicolumn{3}{|l|}{ pT-Kategorie } \\
\hline pT1 & 152 & 9,9 \\
\hline pT2 & 221 & 14,3 \\
\hline pT3 & 960 & 62,3 \\
\hline pT4 & 209 & 13,6 \\
\hline \multicolumn{3}{|l|}{ pN-Kategorie } \\
\hline $\mathrm{pN} 0$ & 973 & 63,1 \\
\hline $\mathrm{pN} 1$ & 372 & 24,1 \\
\hline $\mathrm{pN} 2$ & 197 & 12,8 \\
\hline $\begin{array}{l}\text { Anzahl der untersuchten Lymphknoten, } \\
\text { Median (Range) }\end{array}$ & $32(2-169)$ & \\
\hline Elektivoperation & 1408 & 91,3 \\
\hline Notfalloperation & 134 & 8,7 \\
\hline \multicolumn{3}{|l|}{ Stadium } \\
\hline I & 310 & 20,1 \\
\hline II & 663 & 43,0 \\
\hline III & 569 & 36,9 \\
\hline
\end{tabular}

Bei der spanischen (Lacy) und der amerikanischen Studie (COST) wurden beispielsweise alle Transversumkarzinome ausgeschlossen [26, 27].

Im Rahmen der COST und COLOR-Studien wurden auBerdem alle Patienten mit einem BMI $>30 \mathrm{~kg} / \mathrm{m}^{2}$ oder mit T4-Tumoren (in unserem Krankengut 13,4\%) ausgeschlossen [28].

Der Auschluss von Patienten mit einem BMI $>30 \mathrm{~kg} / \mathrm{m}^{2}$ resultierte daraus, dass in früheren Arbeiten bei der laparoskopischen Chirurgie für dieses Patientenkollektiv deutliche höhere Konversionsraten gezeigt wurden [29-31]. Diese resultieren auch deshalb in schlechteren onkologischen Ergebnissen, da es bei adipösen Patienten bei der laparoskopischen Technik vor Konversion häufiger intraabdominell bei der «Präparateentwicklung» zu unkontrollierten Einrissen des Mesokolons kommt, mit der damit verbundenen schlechteren Präparatequalität. Dies wiederum ist bekanntermaßen mit einer erheblichen Verschlechterung der Prognose verbunden. Im Falle etablierter Lymphknotenmetastasen (UICC III) kommt es dabei zu einer Reduktion des 5-Jahres-Gesamtüberlebens um bis zu 27\% [18].

Neuere Arbeiten zeigen jedoch widersprüchliche Ergebnisse, wonach auch bei Patienten mit einem BMI $>30 \mathrm{~kg} / \mathrm{m}^{2}$ laparoskopische Resektionen ohne erhöhtes Risiko bzw. verschlechterte postoperative Morbidität/Mortalität durchführbar sein sollen $[32,33]$. 
Hinsichtlich des durchschnittlichen BMI sollte erwähnt werden, dass es hier große «regionale» Unterschiede gibt. Im eigenen Krankengut liegt der durchschnittliche BMI bei etwa $27 \mathrm{~kg} / \mathrm{m}^{2}$, im asiatischen Raum jedoch deutlich niedriger (z.B. bei $22 \mathrm{~kg} / \mathrm{m}^{2}$ in Japan) [34].

\section{Wirtschaftlichkeit}

Die Operationsdauer war in allen großen vergleichenden Studien bei den laparoskopischen Resektionen signifikant länger als bei den offenen Resektionen (mediane Differenz von 30-55 $\mathrm{min}$ ) [13].

Die dadurch bedingten Kosten in Verbindung mit zudem erhöhten Materialkosten wurden jedoch durch einen durchschnittlich kürzeren Krankenhausaufenthalt und durch eine geringere Rate an postoperativen Komplikationen ausgeglichen $[13,14]$. Dies spiegelt sich jedoch in den herkömmlichen Entgeltsystemen bisher nicht wider.

Eine andere aktuelle Publikation [35] kommt zu dem Schluss, dass der einzige Umstand, der die Kosteneffektivität der laparoskopischen Chirurgie beeinflusst, die Rate der postoperativen Hernien ist. Aufgrund der zusätzlichen Zeit des Erwerbsausfalls wegen des erforderlichen Hernienverschlusses ist die laparoskopische Resektion nur kosteneffektiv, wenn die Hernienrate geringer oder gleich hoch ist wie bei der offenen Operation. Für alle anderen Variablen bleibt die Laparoskopie weniger kostspielig als die offene Operation, ohne Unterschiede in der Lebensqualität.

In einer von Braga et al. [24] veröffentlichten randomisierten kontrollierten Studie zum Vergleich von offenen und laparoskopischen linksseitigen Kolonresektionen zeigte sich, dass die Dauer des Krankenhausaufenthalts in der offenen Gruppe signifikant länger war (8,7 vs. 7,0 Tage für die Laparoskopie; $p=0,002)$. Die Kosten-Nutzen-Analyse ergab lediglich zusätzliche Kosten von 66 EUR pro laparoskopisch operiertem Patient.

\section{Schlussfolgerungen}

Nach aktueller Datenlage können bei geeigneter Selektion wie auch Expertise des Operateurs Kolonkarzinome mit gleicher onkologischer Qualität laparoskopisch operiert werden. Allerdings gibt es bisher keine einheitlich definierten Kriterien bezüglich Selektion und Expertise.

Die Liste der Ausschlusskriterien ist umfangreich: Karzinome mit Infiltration angrenzender Strukturen oder Organe, Mehrfachkarzinome, Karzinome des Colon transversum mit beiden Flexuren und Notfälle. Auch unter Ausschluss dieser Patientengruppen ist mit einer Konversionsrate in den meisten Kliniken um 20\% zu rechnen; bei sehr erfahrenen laparoskopisch tätigen Chirurgen liegt diese allerdings deutlich niedriger.

Hohe Konversionsraten sind aufgrund schlechterer onkologischer Langzeitergebnisse nicht akzeptabel.

Dies bedeutet, dass ein laparoskopisch versierter Operateur in der Lage sein muss, bei Vorliegen von Ausschlusskriterien für eine laparoskopische Kolonresektion den Eingriff mit gleicher onkologischer Qualität offen durchzuführen, was entsprechende Erfahrung und Lernkurven mit der offenen Technik voraussetzt.

Wegen oft lokal geringer Fallzahlen und wegen der zur Lernkurve erforderlichen hohen Fallzahlen ist zur Erreichung einer hohen onkologischen Qualität (und damit hohen Heilungsraten) eine offene Hemikolektomie außerhalb von spezialisierten Zentren wahrscheinlich vorteilhafter.

Eine «onkologische Gleichwertigkeit» von laparoskopischer und offener Chirurgie resultiert möglicherweise auch daraus, dass in den mittlerweile schon einige Jahre zurückliegenden vergleichenden Studien keine optimierte offene Chirurgie (CME mit CVL) zur Anwendung kam. In den letzten Jahren konnte gezeigt werden, dass die CME mit CVL bei (rechtsseitigen) Kolonkarzinomen zu onkologisch höherwertigen Präparaten und damit besseren onkologischen Langzeitergebnissen führt [16, 19].

\section{Disclosure Statement}

Es bestehen keine Interessenkonflikte seitens der Autoren im Zusammenhang mit dieser Arbeit.

\section{Literatur}

1 Leitlinienprogramm Onkologie (Deutsche Krebsgesellschaft, Deutsche Krebshilfe, AWMF): S3Leitlinie Kolorektales Karzinom, Langversion 1.0, AWMF-Registrierungsnummer: 021-007OL. http:// leitlinienprogramm-onkologie.de/Leitlinien.1.0.html.

2 Kuhry E, Schwenk W, Gaupset R, Romild U, Bonjer HJ: Long-term results of laparoscopic colorectal cancer resection. Cochrane Database Syst Rev 2008;(2):CD003432.
Jayne DG, Thorpe HC, Copeland J, Quirke P, Brown JM, Guillou PJ: Five-year follow-up of the Medical Research Council CLASICC trial of laparoscopically assisted versus open surgery for colorectal cancer. Br J Surg 2010;97:1638-1645.

4 Schmiegel W, Pox C, Reinacher-Schick A, et al: S3Leitlinie «Kolorektales Karzinom», Ergebnisse evidenzbasierter Konsensuskonferenzen am 6./7. Februar 2004 und am 8./9. Juni 2007 (für die Themenkomplexe IV, VI und VII). Z Gastroenterol 2008;46:1-73.
5 Weber K, Merkel S, Perrakis A, Hohenberger W: Is there a disadvantage to radical lymph node dissection in colon cancer? Int J Colorectal Dis 2013; 28:217-226.

6 Merkel S, Schellerer V, Weber K, Göhl J, Hohenberger W: Assessment of advances of outcome quality in colon carcinoma at a single center. Colorectal Cancer 2012;1:215-224.

7 Drolet S, MacLean AR, Myers RP, Shaheen AAM, Dixon E, Buie WD: Elective resection of colon cancer by high-volume surgeons is associated with decreased morbidity and mortality. J Gastrointest Surg 2011;15:541-550. 
8 Kube R, Ptok H, Steinert R, Sahm M, Schmidt U, Gastinger I, Lippert H: Clinical value of laparoscopic surgery for colon cancer. Chirurg 2008;79: $1145-1150$.

$\checkmark 9$ Schlachta CM, Mamazza J, Seshadri PA, Cadeddu M, Gregoire R, Poulin EC: Defining a learning curve for laparoscopic colorectal resections. Dis Colon Rectum 2001;44:217-222.

10 Tekkis PP, Senagore AJ, Delaney CP, Fazio VW: Evaluation of the learning curve in laparoscopic colorectal surgery: comparison of right-sided and left-sided resections. Ann Surg 2005;242:83-91.

11 Robinson CN, Chen GJ, Balentine CJ, Sansgiry S, Marshall CL, Anaya DA, Artinyan A, Albo D, Berger DH: Minimally invasive surgery is underutilized for colon cancer. Ann Surg Oncol 2011;18: 1412-1418.

12 Böttger TC, Mohseni D, Beardi J, Rodehorst A Lernkurve in der laparoskopischen Rektumchirurgie. Zentralbl Chir 2011;136:273-281.

13 Lai JH, Law WL: Laparoscopic surgery for colorectal cancer. Br Med Bull 2012;104:61-89.

-14 Ohtani H, Tamamori Y, Arimoto Y, Nishiguchi Y, Maeda K, Hirakawa K: A meta-analysis of the short- and long-term results of randomized controlled trials that compared laparoscopy-assisted and open colectomy for colon cancer. J Cancer 2012;3: 49-57.

15 Green BL, Marshall HC, Collinson F, Quirke P, Guillou P, Jayne DG, Brown JM: Long-term follow-up of the Medical Research Council CLASICC trial of conventional versus laparoscopically assisted resection in colorectal cancer. $\mathrm{Br} \mathrm{J}$ Surg 2013;100:75-82.

16 Hohenberger W, Weber K, Matzel K, Papadopoulos T, Merkel S: Standardized surgery for colonic cancer: complete mesocolic excision and central ligation - technical notes and outcome. Colorectal Dis 2009;11:354-364, discussion 364-365.

17 Quirke P: Prospective assessment of the quality of surgery in the MRC CLASICC trial: evidence for variation in the plane of surgery in colon cancer, local recurrence and survival. www.ncri.org.uk/ ncriconference/2008abstracts/abstracts/B115.htm.
18 West NP, Morris EJA, Rotimi O, Cairns A, Finan PJ, Quirke P: Pathology grading of colon cancer surgical resection and its association with survival: a retrospective observational study. Lancet Oncol 2008;9:857-865.

19 West NP, Hohenberger W, Weber K, Perrakis A, Finan PJ, Quirke P: Complete mesocolic excision with central vascular ligation produces an oncologically superior specimen compared with standard surgery for carcinoma of the colon. J Clin Oncol 2010;28:272-278.

20 Gouvas N, Pechlivanides G, Zervakis N, Kafousi M, Xynos E: Complete mesocolic excision in colon cancer surgery: a comparison between open and laparoscopic approach. Colorectal Dis 2012;14:1357-1364.

21 Schwenk W, Haase O, Neudecker J, Müller JM: Short term benefits for laparoscopic colorectal resection. Cochrane Database Syst Rev 2005;(3): CD003145.

-22 Noel JK, Fahrbach K, Estok R, Cella C, Frame D, Linz H, Cima RR, Dozois EJ, Senagore AJ: Minimally invasive colorectal resection outcomes: short-term comparison with open procedures. J Am Coll Surg 2007;204:291-307.

23 Janson M, Lindholm E, Anderberg B, Haglind E: Randomized trial of health-related quality of life after open and laparoscopic surgery for colon cancer. Surg Endosc 2007;21:747-753.

24 Braga M, Frasson M, Zuliani W, Vignali A, Pecorelli N, Di Carlo V: Randomized clinical trial of laparoscopic versus open left colonic resection. Br J Surg 2010;97:1180-1186.

25 Hohenberger W, Merkel S: Die laparoskopische Chirurgie des Kolonkarzinoms. Kommentar aufgrund der aktuellen Studienlage. Chirurg 2004;75: 1053-1055.

26 Nelson H, Sargent D, Wieand S, Fleshman J, Anvari M, Stryker SJ, Beart R Jr, Hellinger M, Flanagan R, Peters W, Ota D: A comparison of laparoscopically assisted and open colectomy for colon cancer. N Engl J Med 2004;350:2050-2059.
27 Lacy AM, García-Valdecasas JC, Delgado S, Castells A, Taurá P, Piqué JM, Visa J: Laparoscopyassisted colectomy versus open colectomy for treatment of non-metastatic colon cancer: a randomised trial. Lancet 2002;359:2224-2229.

28 Bozetti F: Open colectomies vs. laparoscopic colectomies: can we generalize to real-world practice settings? Dis Colon Rectum 2009;52:755-756.

29 Senagore A: Laparoscopic colectomy in obese and nonobese patients. J Gastrointest Surg 2003;7:558561.

30 Pandya S, Murray JJ, Coller JA, Rusin LC: Laparoscopic colectomy: indications for conversion to laparotomy. Arch Surg 1999;134:471-475.

31 Pikarsky AJ, Saida Y, Yamaguchi T, Martinez S, Chen W, Weiss EG, Nogueras JJ, Wexner SD: Is obesity a high-risk factor for laparoscopic colorectal surgery? Surg Endosc 2002;16:855-858.

32 Poulsen M, Ovesen H: Is laparoscopic colorectal cancer surgery in obese patients associated with an increased risk? Short-term results from a single center study of 425 patients. J Gastrointest Surg 2012;16:1554-1558.

33 Feroci F, Baraghini M, Lenzi E, Garzi A, Vannucchi A, Cantafio S, Scatizzi M: Laparoscopic surgery improves postoperative outcomes in high-risk patients with colorectal cancer. Surg Endosc 2012;27: 1130-1137.

34 West NP, Kobayashi H, Takahashi K, Perrakis A Weber K, Hohenberger W, Sugihara K, Quirke P: Understanding optimal colonic cancer surgery: comparison of Japanese D3 resection and European complete mesocolic excision with central vascular ligation. J Clin Oncol 2012;30:1763-1769.

35 Jensen CC, Prasad LM, Abcarian H: Cost-effectiveness of laparoscopic vs open resection for colon and rectal cancer. Dis Colon Rectum 2012;55:10171023 